\title{
ESTUDO DO EFEITO DAS CONDIÇÕES CLIMÁTICAS NO COMPORTAMENTO TÉRMICO DO FRUTO DE JATOBÁ DE DUAS SAFRAS
}

\section{STUDY OF THE CLIMATE EFFECT CONDITIONS IN THE THERMAL BEHAVIOR OF TWO CROPS OF THE JATOBÁ FRUIT}

\section{Natalia Raiz Segismundo}

Mestre em Ciências/Universidade Federal de São Paulo - UNIFESP. Doutoranda do Programa de Pós-Graduação em Biologia Química da UNIFESP - Campus Diadema. (natyraiz@gmail.com)

\section{Jivaldo do Rosário Matos}

Doutor em Química Inorgânica/Universidade de São Paulo - USP. Livre Docente (Química Analítica) do Instituto de Química/USP. (jdrmatos@gmail.com)

\section{Lucildes Pita Mercuri}

Doutora em Química (Química Analítica)/USP. Universidade Federal de São Paulo - UNIFESP. (Ipmercuri@gmail.com)

\section{Resumo}

O fruto de jatobá usado como alimento apresenta propriedades que auxiliam na prevenção de doenças crônicas. O estudo da influência das condições climáticas na decomposição térmica do fruto foi proposto para avaliar, com base em suas características químicas, sua possível aplicação no setor farmacêutico. Neste trabalho, serão analisados frutos das safras de 2010 e 2012 . O fruto de jatobá se mostrou uma matriz complexa para a caracterização química, físico-química e termoanalítica. As curvas termogravimétricas evidenciaram que a decomposição térmica de todas as amostras é completa a $900^{\circ} \mathrm{C}$. Observou-se que as amostras de casca lavada de 2012 (CASL12), casca não lavada de 2012 (CASNL12) e semente de 2012 (SEM12) perderam algumas bandas em relação a 2010, indicando que as condições climáticas afetaram os frutos. Com relação a todas as amostras estudadas, a fibra de 2012 (FIB12) e a SEM12 apresentaram maiores teores de cinza e umidade, respectivamente. Porém, as amostras de polpa da safra de 2012 e fibra apresentaram quatro eventos de decomposição térmica, diferindo da safra de 2010 . As condições climáticas das safras precedentes se mostraram muito diferentes e isso afetou consideravelmente o comportamento térmico dos frutos de jatobá.

Palavras-chave: Condições climáticas, comportamento térmico, TG/DTG

\section{Abstract}

The fruit of jatoba besides being part of the food class presentations that help in the prevention of chronic diseases. The study of the influence of the climatic conditions on the thermal decomposition of the fruit was proposed to evaluate, based on its chemical characteristics, its possible application in different industrial sectors, such as: food and pharmaceutical. This work will analyze the fruits of the 2010 and 2012 harvests. The fruit of jatoba showed to be a complex matrix for the chemical, physicochemical and thermochemical characterization. The thermogravimetric curves showed that the thermal decomposition of all the samples is complete at $900^{\circ} \mathrm{C}$. It was observed that the washed rind samples 2012 (CASL12), not washed rind 2012 (CASNL12) and seed 2012 (SEM12) lost some bands compared to 2010, indicating that the weather affected the fruit. In relation to all the studied samples, the fiber of 2012 (FIB12) and SEM12 presented higher levels of ash and humidity, respectively. However, the pulp samples from the 2012 harvest and fiber presented four thermal decomposition events, differing from the 2010 harvest. The climatic conditions of the previous harvests were very different and this affected considerably the thermal behavior of the jatoba fruits.

Keywords: Climatic conditions, thermal behavior, TG / DTG

\section{INTRODUÇÃO}

O jatobá (Hymenaea courbaril L.) é uma
planta da família

Caesalpinioideae que apresenta nomes diferentes dependendo da região, como jatobá, jutaí, jutaí-açu, jutaí-bravo, jutaí-grande, jataí, jataí-açu, jataí-grande, dentre outros. O fruto é 
uma vagem indeiscente, lenhosa, glabra, oblonga a cilíndrica, que mede $8-15 \mathrm{~cm}$ de comprimento, sua casca é espessa e vermelhoescura, e a polpa é farinácea, com gosto adocicado e amarelo-claro. As sementes, em número de 2 a 6 por fruto ou mais, apresentam formato obovóide a elipsóide, 1,8-2,8 cm de comprimento, 1,4-2,0 cm de largura, 0,8-1,4 cm de espessura e pesam 2,1-6,2 g A fibra é pétrea, lisa e pardo-clara a pardo-escuro (DE MELO; MENDES, 2005; SILVA et al. 2001; DE MELO; MENDONÇA; MENDES, 2004).

O jatobá pode fornecer uma farinha que apresenta um elevado conteúdo de fibra alimentar total, sendo seu consumo importante para os seres humanos e na prevenção de doenças crônicas. A fibra alimentar apresenta diversos efeitos fisiológicos, como alteração nas funções gastrointestinais e redução dos níveis de colesterol, glicemia e insulina pós-prandial. Esta farinha pode ser misturada com a farinha de trigo para a fabricação de biscoitos, snacks e cookies. O extrato da casca de Jatobá, que é utilizada na Amazônia, apresenta substâncias que combatem a fadiga e pode ser utilizado como suplemento alimentar. $\mathrm{O}$ fruto contém, ainda, terpenóides ou compostos fenólicos que possuem atividade antibactericida (SASAKI et al., 2009).

As sementes deste fruto contêm xiloglucanas e galactomananas que são as principais hemiceluloses presentes na parede celular de dicotiledôneas, utilizadas na fabricação de papéis (MATUDA; MARIA NETO, 2005), e as galactoxiloglucanas funcionam como polissacarídeos de armazenamento (FREITAS et al., 2004). Lucyszyn e colaboradores (2009) relatam que xiloglucanas extraídas da semente, ao serem modificadas, podem ser utilizadas na encapsulação de fármacos.

O chá da polpa do fruto apresenta procianidinas (taninos condensados) oligoméricas que têm efeitos benéficos para encefalomielite autoimune experimental e reduz os sintomas de artrite em ratos (MIYAKE et al., 2008). O extrato metanóico da casca de jatobádo-cerrado (Hymenaea stigonocarpa Mart. ex Hayne) apresenta atividade antidiarreica, cicatrizante e gastro-protetora (FARIAS et al., 2017). A dieta com a polpa do fruto demonstrou efeitos curativos de úlceras peptídicas (ORSI et al., 2012).

Tendo em vista o grande potencial de aplicação do fruto de jatobá, este trabalho tem como objetivo principal estudar o efeito das condições climáticas na região da coleta sob o comportamento térmico do fruto de jatobá.

\section{METODOLOGIA}

\subsection{Material}

Neste estudo, foram utilizadas amostras de frutos de jatobá das safras de 2010 e 2012 obtidos, por doação, de uma fazenda na região de Franca - SP.

\subsection{Métodos}

\subsubsection{Procedimento de coleta}

Os frutos foram coletados diretamente da mesma árvore nos meses de setembro de 2010 e 2012. As condições climáticas mensuradas na estação de coleta de dados meteorológicos de Franca (Latitude: $-20.58^{\circ}$, Longitude: $-47.36^{\circ} \mathrm{e}$ Altitude: $1026.20 \mathrm{~m}$ ), do Instituto Nacional de Meteorologia (INMET, 2017), encontram-se na Tabela 1. Os frutos foram armazenados em sacos de ráfia e transportados dentro de caixas de papelão até serem utilizados para estudo.

\subsubsection{Preparação da amostra}

Foram utilizados quinze frutos para a obtenção das amostras. Os frutos foram divididos em: semente (SEM), polpa (POL), fibras (FIB), casca lavada (CASL) e casca não lavada (CASNL). A casca foi submetida a uma lavagem com água destilada para a remoção de resíduos de polpa e fibra, e foi seca à temperatura ambiente. Com intuito de comparação, foram realizados os ensaios com a casca não lavada. Com exceção da POL, todas as amostras foram processadas em liquidificador, para aumentar as superfícies de contato. Também, com exceção da POL, todas as amostras trituradas foram submetidas à peneiração (peneira 60 mesh), com o intuito de homogeneizar o tamanho de partícula desses materiais.

\subsubsection{Análise Elementar (AE)}

Os teores percentuais de carbono, hidrogênio e nitrogênio das amostras foram determinados na Central Analítica do Instituto de Química da Universidade de São Paulo, utilizando um equipamento Elemental Analysis, modelo 2400, da marca Perkin Elmer. O 
Tabela 1. Condições climáticas dos meses de setembro de 2010 e 2012 para a região de Franca-SP

\begin{tabular}{c|c|c|c|c}
\hline \multirow{2}{*}{ Ano } & \multicolumn{4}{|c}{ Médias mensais } \\
\cline { 2 - 5 } & $\begin{array}{c}\text { Precipitação } \\
\text { Total / mm }\end{array}$ & $\begin{array}{c}\text { Número de } \\
\text { Dias de } \\
\text { Precipitação }\end{array}$ & $\begin{array}{c}\text { Precipitação } \\
\text { Média / mm }\end{array}$ & $\begin{array}{c}\text { Umidade } \\
\text { Relativa } \\
\text { Média / \% }\end{array}$ \\
\hline $\mathbf{2 0 1 0}$ & 101,0 & 8 & 3,37 & 53,1 \\
\hline $\mathbf{2 0 1 2}$ & 85,9 & 5 & 2,86 & 50,3 \\
\hline \multicolumn{2}{|c|}{ Fonte: Dados da Rede do INMET (2017). } \\
\hline
\end{tabular}

2.2.4. Espectroscopia de absorção na região do infravermelho com transformada de Fourier (FTIR)

Os espectros de FTIR foram obtidos por um espectrômetro de absorção da marca BOMEM, modelo MB102, na região de 4000 a $400 \mathrm{~cm}-1$ e registrados como transmitância vs número de ondas. As amostras foram trituradas, dispersas em $\mathrm{KBr}$, prensadas para formação de pastilhas e adaptadas ao porta amostra para registro dos espectros. Estes ensaios foram realizados na Central Analítica do Instituto de Química da Universidade de São Paulo.

\subsubsection{Termogravimetria/termogravimetri a derivada (TG/DTG)}

As curvas TG/DTG foram obtidas utilizando uma termobalança TGA-51 (Shimadzu), com cadinho de Pt, massa de amostra $\approx 50 \mathrm{mg}$, sob atmosfera dinâmica de ar (50 $\left.\mathrm{mL} \mathrm{min}^{-1}\right)$, razão de aquecimento de $10^{\circ} \mathrm{C} \mathrm{min}^{-1} \mathrm{e}$ faixa de temperatura entre temperatura ambiente $\left(\mathrm{T}_{\mathrm{amb}}\right)$ a $900^{\circ} \mathrm{C}$.

\subsubsection{Calorimetria Exploratória Diferencial (DSC)}

As curvas DSC foram obtidas utilizando uma célula calorimétrica, modelo DSC-50, da marca Shimadzu, sob atmosfera dinâmica de $\mathrm{N}_{2}\left(100 \mathrm{~mL} \mathrm{~min}^{-1}\right)$ e razão de aquecimento de $10{ }^{\circ} \mathrm{C} \mathrm{min}^{-1}$, no intervalo de temperatura de $\mathrm{T}_{\mathrm{amb}}$ a $550{ }^{\circ} \mathrm{C}$, em cápsulas de alumínio parcialmente fechadas e massa de amostra de, aproximadamente, $2 \mathrm{mg}$.

\section{RESULTADOS E DISCUSSÃO}

\subsection{Análise Elementar ( $A E)$}

Os resultados de $A E$ mostram a porcentagem de carbono $(C)$, nitrogênio $(N)$ e hidrogênio $(H)$ presentes nas amostras. $A$ Tabela 2 ilustra as porcentagens desses elementos nas amostras CASL, CASNL, FIB, POL e SEM dos frutos de jatobá da safra de 2010 e de 2012. Foi realizada apenas uma análise de cada amostra.

A partir destes resultados, pode-se predizer que o teor de $\mathrm{H}$ e $\mathrm{N}$ das amostras da safra de 2010 apresenta valores similares entre as amostras, enquanto que o teor $C$ apresentou maior variação entre as mesmas. Em ambas as safras, o maior teor de C observado foi em CASNL. Este teor pode ser associado com a presença de resíduos de polpa e fibra que, em CASL, foram retirados pela lavagem realizada. Pode-se observar um aumento dos teores dos três elementos nas amostras CASL e CASNL na safra de 2012 em relação às amostras da safra de 2010, o que pode estar associado com a disponibilidade de nutrientes no solo.

A amostra de FIB apresentou a menor porcentagem de $\mathrm{C}$ e $\mathrm{H}$, tanto em 2010 quanto em 2012, o que está associado com as características da amostra, tais como a presença de compostos oxigenados (por exemplo, polímeros orgânicos $-\mathrm{C}_{x} \mathrm{H}_{\mathrm{y}} \mathrm{O}_{z}$ ). No entanto, a porcentagem de $\mathrm{N}$ é maior na safra de 2012, provavelmente devido à maior disponibilidade de nutrientes do solo. $\mathrm{Na}$ amostra de POL, a porcentagem de $\mathrm{C} \mathrm{e}$ $\mathrm{H}$ é similar entre as safras, porém, observa- 
se que na safra de 2012 tem-se uma \% de $\mathrm{N}$ maior. A SEM evidenciou teor de $\mathrm{C}$ intermediário devido à presença de compostos inorgânicos nas safras de 2010.
Pode-se sugerir que estes compostos estejam presentes com base na característica física da amostra, tal como a dureza.

Tabela 2. Resultados de AE para as amostras de frutos de jatobá das safras de 2010 e 2012

\begin{tabular}{c|c|c|c|c|c|c}
\hline \multirow{2}{*}{ Amostra } & \multicolumn{3}{|c|}{2010} & \multicolumn{3}{c}{$\mathbf{2 0 1 2}$} \\
\cline { 2 - 7 } & $\mathbf{C} / \%$ & $\mathbf{H} / \%$ & $\mathbf{N} / \%$ & $\mathbf{C} / \%$ & $\mathbf{H} / \%$ & $\mathbf{N} / \%$ \\
\hline CASL & 49,3 & 6,4 & 0,3 & 54,6 & 7,3 & 0,6 \\
\hline CASNL & 54,3 & 6,4 & 0,4 & 56,9 & 7,5 & 0,5 \\
\hline FIB & 39,6 & 5,7 & 0,4 & 39,2 & 5,9 & 1,1 \\
\hline POL & 40,0 & 6,1 & 0,7 & 40,8 & 6,3 & 1,3 \\
\hline SEM & 43,2 & 6,2 & 0,8 & 44,4 & 6,2 & 0,9 \\
\hline
\end{tabular}

Fonte: Autores.

3.2. Espectroscopia de absorção na região do infravermelho com transformada de Fourier (FTIR)

Os espectros das amostras de CASL e CASNL das safras de 2010 [Fig. 1(a)] e 2012 [Fig. 1(b)] apresentaram perfil similar; foram identificadas bandas características de carbonatos, carboidratos e lipídeos. Observou que algumas bandas não estavam presentes na safra de 2012, o que indica que condições ambientais afetaram a composição química dessas amostras.

Nas amostras de FIB e POL das duas safras apresentam perfil similar e foi possível identificar bandas características de celulose. Diferentemente das amostras de casca, não foi possível identificar a perda de bandas entre as safras na fibra, porém houve mudança na intensidade e na largura de algumas bandas.

O espectro da amostra de SEM da safra de 2012 apresentou perda de duas bandas, sugerindo, na safra de 2010, a presença de $\mathrm{H}_{2} \mathrm{O}$. Em ambas as safras, se propõem a presença de bandas características de óleos vegetais.

Pode-se sugerir que a polpa e a fibra auxiliem na melhora da constipação intestinal devido à presença da celulose, e os óleos vegetais encontrados nas sementes podem ser promissores para a produção de biodiesel ou na alimentação.

3.3. Termogravimetria/termogravimetria derivada (TG/DTG) e Calorimetria Exploratória Diferencial (DSC)

\subsubsection{Casca Lavada (CASL)}

As curvas TG/DTG das amostras das safras de 2010 e 2012 (Figura 2) mostram que a decomposição térmica ocorre em vários eventos consecutivos e alguns concomitantes de perda de massa. Estes eventos foram agrupados em três faixas de temperatura. $\mathrm{O} 1^{\circ}$ evento é associado à desidratação da amostra e à liberação de compostos voláteis presentes. Os $2^{\circ}$ e $3^{\circ}$ eventos térmicos equivalem à decomposição térmica do material orgânico presente $e$ à liberação do material carbonáceo. A porcentagem de resíduo obtido equivale ao teor de sais minerais (cinzas) presente na amostra.

Observa-se que a amostra da safra de 2012 apresenta maior porcentagem de perda de massa no $1^{\circ}$ evento de decomposição térmica, indicando que essa amostra absorveu maior quantidade de água do que a amostra de 2010. No $2^{\circ}$ evento, porém, ocorre uma diminuição da perda de massa em $3 \%$, o que pode ser devido à ausência das bandas verificadas nos espectros de FTIR.

As curvas DSC das duas amostras (Figura 2) apresentam perfil térmico similar, sendo que $01^{\circ}$ evento é inerente à desidratação térmica da amostra e a decomposição da matéria orgânica apresenta perfil exotérmico. $\mathrm{Na}$ amostra de 2010, o $1^{\circ}$ evento apresenta $\Delta \mathrm{H}=-250,35$ $\mathrm{J} \mathrm{g}^{-1}$, enquanto que na safra de $2012 \circ \Delta \mathrm{H}$ $=-239,61 \mathrm{~J} \mathrm{~g}^{-1}$. Os dados das curvas TG/DTG indicaram que a amostra de 2012 
apresentou maior teor de água, sendo, por isso, esperado que o $\Delta \mathrm{H}$ fosse maior do que o da safra de 2010, porém, não foi o observado, provavelmente, devido a não presença das bandas, como evidenciado no espectro de FTIR.

Figura 1. Espectros de FTIR das amostras de CASNL, CASL, FIB, POL e SEM das safras de 2010 (a) e 2012 (b)
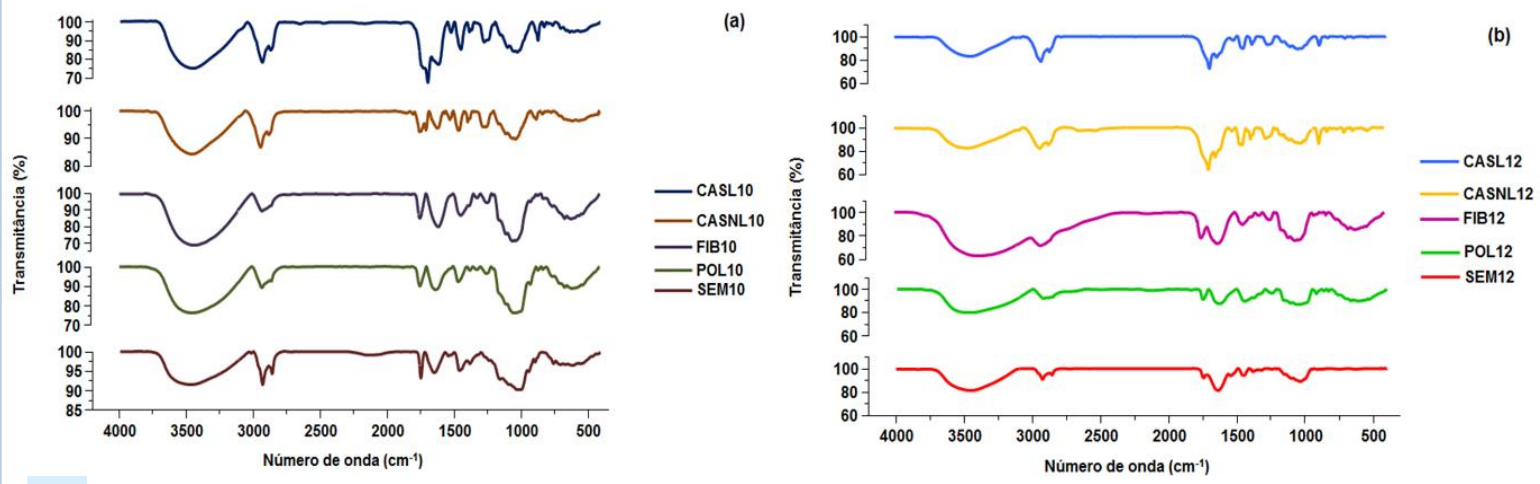

Fonte: Autores.

Figura 2. Curvas TG/DTG e DSC da amostra de CASL das safras de 2010 e 2012

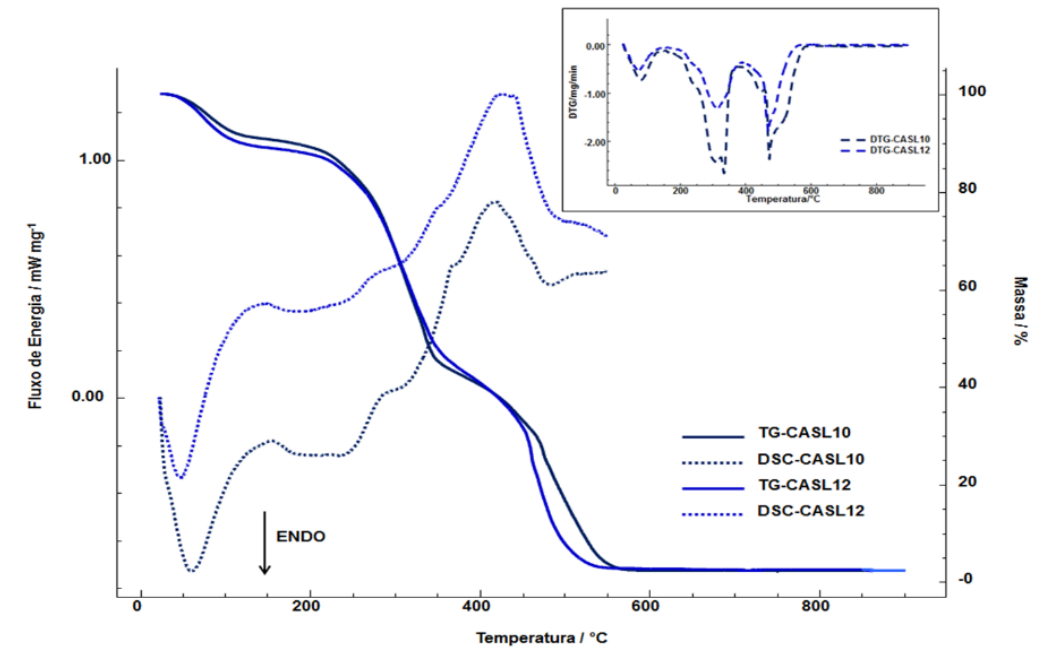

Fonte: Autores.

3.3.2. Casca não lavada (CASNL)

Analogamente às amostras de CASL, as curvas TG/DTG das amostras de CASNL de ambas as safras apresentam decomposição térmica em três eventos consecutivos (Figura 3 ). $\mathrm{O} 1^{\circ}$ evento tratase da desidratação térmica e da liberação de compostos voláteis presentes, $02^{\circ}$ evento corresponde à formação do material carbonáceo e $03^{\circ}$ à liberação do material carbonáceo formado anteriormente. O resíduo coletado na temperatura de $900{ }^{\circ} \mathrm{C}$ corresponde à quantidade de sais minerais presentes nas amostras.
Diferentemente da amostra de CASL, - perfil de decomposição térmica da amostra de CASNL entre as safras é distinto. Destaca-se a diferença entre a porcentagem do $1^{\circ}$ evento entre as amostras, indicando que a amostra de 2012 apresenta uma quantidade de água e/ou voláteis menor do que na amostra de 2010, o que é confirmado pela ausência da banda de FTIR de estiramento $\mathrm{C}=\mathrm{O}$ de éster na safra de 2012. No $2^{\circ}$ evento, a amostra de 2012 apresentou uma porcentagem de $2 \%$ menor que a safra de 2010, possivelmente devido à perda das 
bandas de deformação $\mathrm{CH}_{3}$ e N-H na safra mais recente, verificado pela técnica de FTIR.

As curvas DSC das amostras de cascas não lavada (Figura 3) apresentaram perfil de decomposição térmica diferente, indicando que houve interferência externa, como observada pelas curvas TG/DTG.
Nota-se que $01^{\circ}$ evento de decomposição térmica, na amostra de 2010, apresenta perfil endotérmico com $\Delta \mathrm{H}$ de $-281,33 \mathrm{~J} \mathrm{~g}^{-1}$, enquanto na de $2012 \mathrm{com} \Delta \mathrm{H}=-164,21 \mathrm{~J} \mathrm{~g}$ 1. Esta amostra apresentou um valor de entalpia, para o $1^{\circ}$ evento, menor do que a amostra de 2010, corroborando com os dados de TG/DTG.

Figura 3. Curvas TG/DTG e DSC da amostra de CASNL das safras de 2010 e 2012

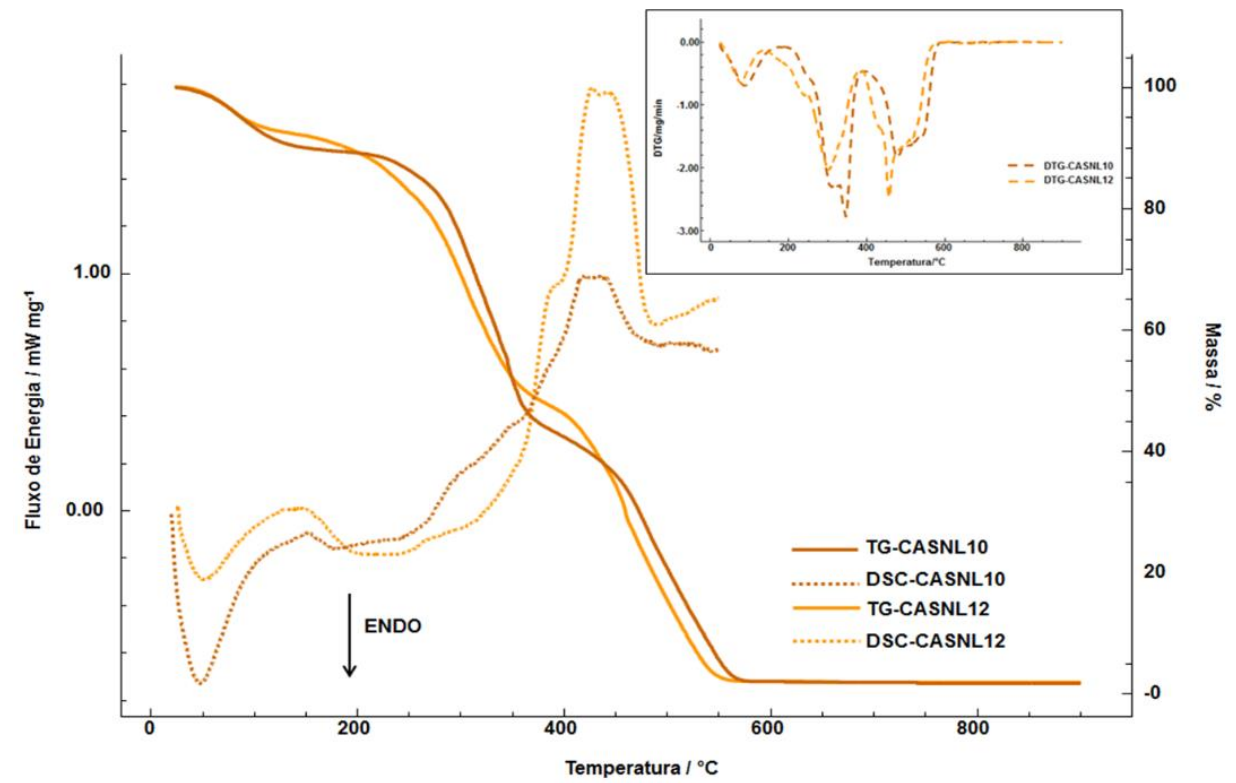

Fonte: Autores.

\subsubsection{Fibra (FIB)}

As curvas TG/DTG das amostras de fibra (FIB) das safras estudadas (Figura 4) evidenciaram que a decomposição térmica ocorre em vários eventos consecutivos, sendo que $01^{\circ}$ evento refere-se à desidratação da amostra e $\circ 2^{\circ}$ e $\circ 3^{\circ}$ eventos à decomposição térmica do material orgânico. A amostra de 2012 apresentou um $4^{\circ}$ evento, compreendido em $\quad 551-900^{\circ} \mathrm{C}, \quad$ característico da decomposição de material inorgânico. A porcentagem de massa obtida na temperatura de $900^{\circ} \mathrm{C}$ corresponde ao resíduo que é composto de material inorgânico presente nesta amostra, visto que é estável a partir de $700^{\circ} \mathrm{C}$.

Como apresentado nos dados de $\mathrm{AE}$, a FIB é formada por uma maior quantidade de compostos oxigenados. A porcentagem residual observada para a fibra (a maior entre as cinco amostras) pode ser relacionada com a formação de carbonatos (a partir da decomposição térmica dos compostos oxigenados), somada à presença de óxidos e/ou hidróxidos metálicos formados no processo de decomposição.

As curvas DSC (Figura 4) das duas safras apresentam perfil similar. $O 1^{\circ}$ evento é endotérmico e refere-se à desidratação da amostra. Para a safra de 2010, a entalpia desse evento foi de $251,66 \mathrm{~J} \mathrm{~g}-1$ e, para a amostra de 2012, foi de $-163,23 \quad \mathrm{~J} \mathrm{~g}^{-1}$. Observou-se que a entalpia de desidratação da safra de 2012 foi menor do que a de 2010, resultado este esperado, visto que a amostra mais recente apresentou uma porcentagem de perda de massa nas curvas TG/DTG menor do que em 2010. 
Figura 4. Curvas TG/DTG e DSC da amostra de FIB das safras de 2010 e 2012

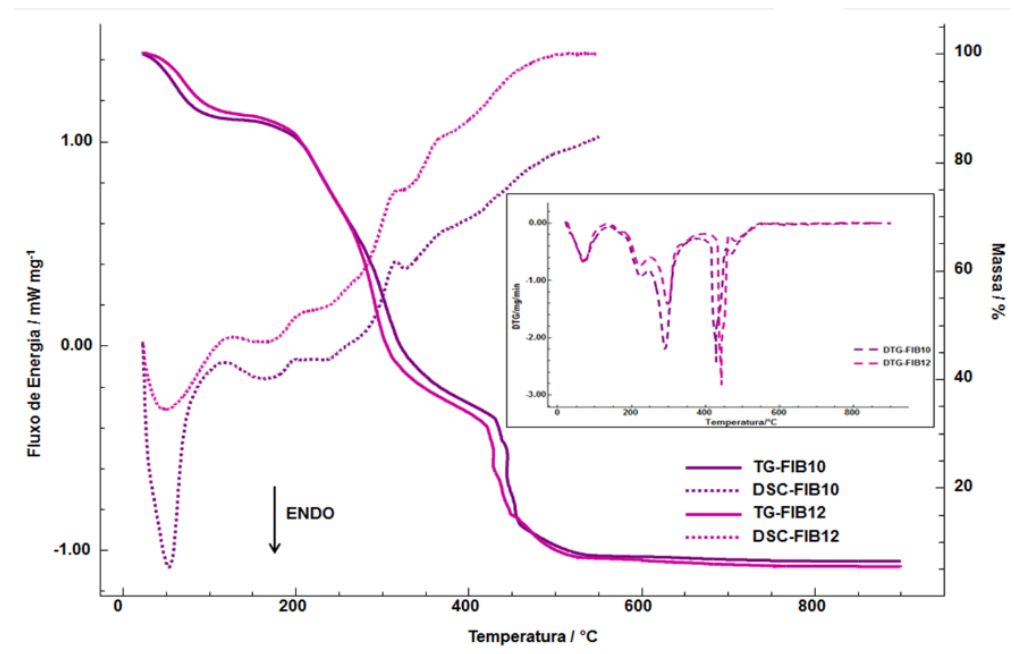

Fonte: Autores.

\subsubsection{Polpa (POL)}

A Figura 5 representa as curvas TG/DTG da amostra de POL. A decomposição térmica ocorre em eventos concomitantes e consecutivos, ou seja, o $1^{\circ}$ evento, analogamente aos anteriores, é relativo à liberação de compostos voláteis e à desidratação da amostra. Contudo, o $2^{\circ} \mathrm{e}$ $3^{\text {o }}$ eventos equivalem à formação de material carbonáceo, e o $4^{\circ}$ evento é referente à liberação do material carbonáceo. No entanto, para amostra da safra de 2010, o 5o evento, que ocorre entre 566 a $900^{\circ} \mathrm{C}$, é equivalente à decomposição térmica de um tipo de material inorgânico presente nessa amostra.

Diferentemente das outras amostras, a formação do material carbonáceo ocorreu em duas etapas, o que, provavelmente, está associado com a presença de alguns compostos que podem se decompor em temperaturas mais altas, fato este que caracteriza o $3^{\circ}$ evento térmico evidenciado nas curvas. A porcentagem de resíduo obtido a $900^{\circ} \mathrm{C}$ é correspondente ao teor de sais minerais presente nesta amostra.

As curvas DSC das amostras de POL estão representadas na Figura 5 . A amostra de 2010 apresentou vários eventos, sendo eles consecutivos e concomitantes. O $1^{\circ}$ evento tem perfil endotérmico e refere-se à desidratação térmica $\left(\Delta \mathrm{H}=-249,13 \mathrm{~J} \mathrm{~g}^{-1}\right)$. A amostra de
POL de 2012 apresentou um evento endotérmico, característico da desidratação, com entalpia $(\Delta \mathrm{H})$ de $198,25 \mathrm{~J} \mathrm{~g}^{-1}$. Analogamente às amostras de CASNL e FIB, a amostra da safra de 2012 apresentou um valor de entalpia para 0 evento de desidratação menor do que a de 2010, confirmando os dados de TG/DTG.

\subsubsection{Semente (SEM)}

A Figura 6 mostra as curvas TG/DTG das amostras de semente das safras de 2010 e 2012. Nessas curvas, fica evidente a presença de eventos consecutivos e alguns concomitantes de decomposição térmica, ou seja, o $1^{\circ}$ evento é devido à liberação de compostos voláteis e à desidratação térmica da amostra, o $2^{\circ}$ evento é responsável pela formação de material carbonáceo e o $3^{\circ}$ evento refere-se à liberação do material carbonáceo. A porcentagem de resíduo obtida a $900{ }^{\circ} \mathrm{C}$ é correspondente ao teor de cinzas (sais minerais) na amostra.

Para a amostra da safra de 2010, a hipótese de liberação de $\mathrm{H}_{2} \mathrm{O}$ no $1^{\circ}$ evento de decomposição térmica é corroborada pela presença das bandas características de água evidenciadas pela técnica de FTIR (3460, 2132 e $1649 \mathrm{~cm}^{-1}$ ), enquanto que a safra de 2012 apresentou uma pequena diminuição da perda de massa no $1^{\circ}$ evento, em comparação com 2010, o que pode estar associado às perdas das 
Figura 5. Curvas TG/DTG e DSC da amostra de POL das safras de 2010 e 2012

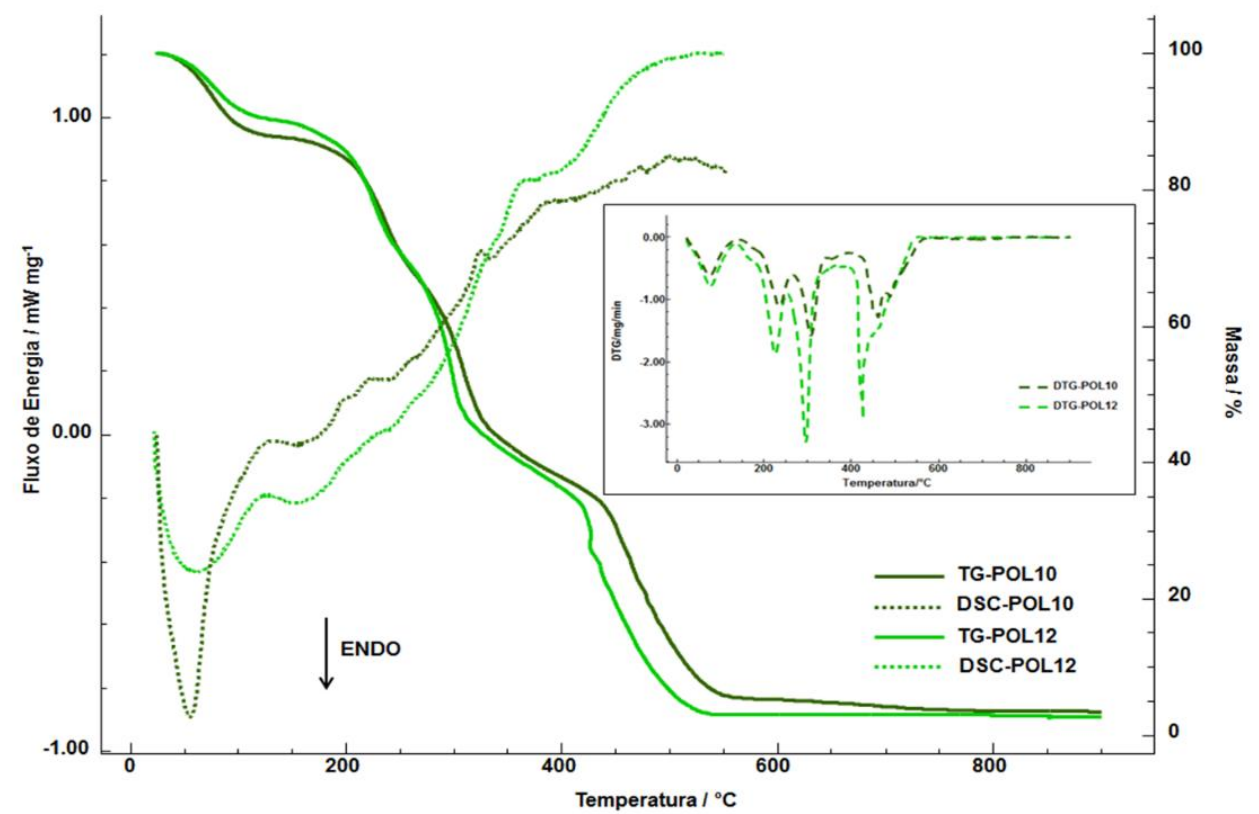

Fonte: Autores.

Figura 6. Curvas TG/DTG e DSC da amostra de SEM das safras de 2010 e 2012

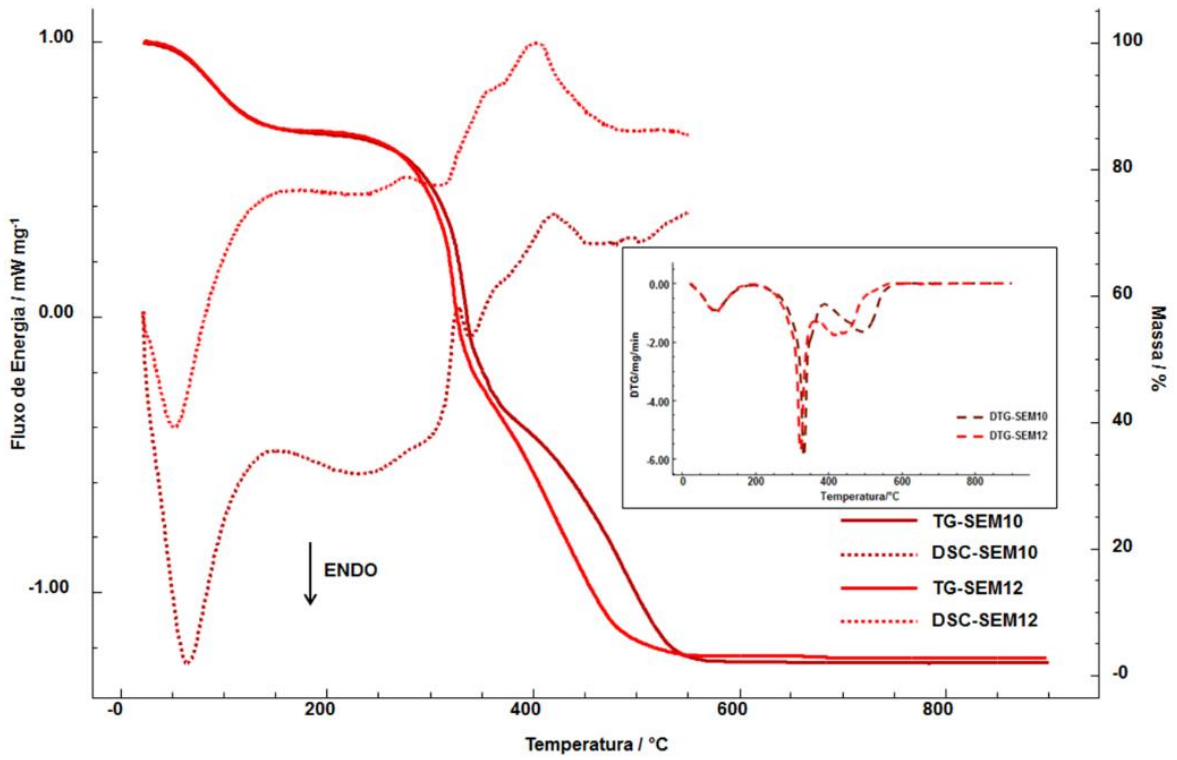

Fonte: Autores.

A Figura 6 apresenta as curvas DSC das amostras de SEM das duas safras estudadas. A amostra SEM10 exibiu um perfil exotérmico de decomposição térmica, contudo, $01^{\circ}$ evento é endotérmico, caracterizando a desidratação $(\Delta \mathrm{H}=$ $\left.439,34 \mathrm{~J} \mathrm{~g}^{-1}\right)$. Análoga à amostra de 2010, a amostra SEM12 apresenta um evento endotérmico, referente à desidratação, com $\Delta \mathrm{H}$ de $-282,64 \mathrm{~J} \mathrm{~g}^{-1}$, e, sequencialmente, 
observaram-se eventos exotérmicos caracterizando a decomposição térmica da amostra. A entalpia desta amostra é menor do que a amostra de 2010, corroborando com os dados de TG/DTG.

\subsection{Considerações sobre as condições climáticas}

Devido às diferenças do comportamento térmico das amostras entre as duas safras, verificou-se a necessidade de investigar mais detalhadamente sobre as condições climáticas da região de coleta. Essa observação condiz com a única variável, já que os frutos coletados nas duas safras são da mesma época e da mesma árvore. Os dados das condições climáticas foram retirados dos dados da rede de estações do Instituto Nacional de Meteorologia (INMET). A estação escolhida foi a da região de Franca-SP, localizada na latitude $-20,58^{\circ}$, longitude $-47,36^{\circ}$ e altitude de $1026,20 \mathrm{~m}$.

Para uma melhor avaliação da interferência das condições ambientais nos frutos, escolheram-se os dados a partir de janeiro de 2009 até dezembro de 2012. Na Tabela 3, encontram-se os dados das médias anuais da precipitação total, número de dias de precipitação, precipitação média e umidade relativa média, dados importantes a serem avaliados.

Tabela 3. Médias anuais da precipitação total, número de dias de precipitação, precipitação média e umidade relativa média da estação de Franca-SP nos anos de 2009 a 2012

\begin{tabular}{c|c|c|c|c}
\hline \multirow{2}{*}{ Ano } & \multicolumn{4}{|c}{ Médias Anuais } \\
\cline { 2 - 5 } & $\begin{array}{c}\text { Precipitação } \\
\text { Total/mm }\end{array}$ & $\begin{array}{c}\text { Número de } \\
\text { Dias de } \\
\text { Precipitação }\end{array}$ & $\begin{array}{c}\text { Precipitação } \\
\text { Média/mm }\end{array}$ & $\begin{array}{c}\text { Umidade } \\
\text { Relativa } \\
\text { Média/\% }\end{array}$ \\
\hline $\mathbf{2 0 0 9}$ & 169,7 & 14 & 5,58 & 73,3 \\
$\mathbf{2 0 1 0}$ & 135,7 & 11 & 4,47 & 66,0 \\
$\mathbf{2 0 1 1}$ & 135,7 & 12 & 4,45 & 68,4 \\
$\mathbf{2 0 1 2}$ & 128,2 & 10 & 4,14 & 67,0 \\
\hline Fonte:
\end{tabular}

A partir destes dados, pode-se inferir que 0 ano antecedente da safra de 2010 apresentou maior precipitação e umidade relativa em relação ao ano de 2011, provando que os frutos coletados em 2012 deverão exibir um teor de água menor do que o de 2010, como se verificou pelas curvas TG/DTG. Estudos mostraram que o aumento da temperatura regional e a diminuição da disponibilidade de água afetam o crescimento da planta de $H$. courbaril (LOCOSSELLI et al., 2016; DO NASCIMENTO et al., 2011), portanto, o crescimento do fruto também será afetado e, por consequência, a sua composição química será diferente. Esse fator de precipitação também afeta a disponibilidade de nutrientes e o metabolismo da planta (LOCOSSELLI et al., 2016; DO NASCIMENTO et al., 2011), o que justifica o porquê do comportamento térmico de algumas amostras apresentarem diferenças entre as safras estudadas.

\section{CONCLUSÃO}

Observou-se que as amostras de CASL12, CASNL12 e SEM12 perderam bandas em comparação com as amostras de 2010. Quando se comparam todas as amostras estudadas, a fibra e a semente da safra de 2012 apresentaram maiores teores de cinza e umidade, respectivamente. Contudo, nas curvas TG/DTG das amostras de polpa e fibra de 2012, verificaram-se quatro eventos de decomposição térmica, diferindo da safra de 2010. O fruto de jatobá se mostrou uma matriz complexa para a caracterização química, físico-química e termoanalítica. As curvas TG/DTG evidenciaram que a decomposição térmica de todas as amostras é completa a $900^{\circ} \mathrm{C}$. As condições 
climáticas das safras precedentes se mostraram muito diferentes e isso afetou, consideravelmente, o comportamento térmico dos frutos de jatobá.

\section{AGRADECIMENTOS}

Aos órgãos de fomento CAPES, CNPq e FAPESP e ao Sr. Elder Moscardini pela doação dos frutos de jatobá para este estudo.

\section{REFERÊNCIAS}

DE MELO, M. G. G.; MENDES, A. M. S. Jatobá Hymenaea courbaril L. Informativo Técnico Rede de Sementes da Amazônia, v. 9, 2005.

MENDONÇA, M. S.; MENDES, A. M. S. Análise morfológica de sementes, germinação e plântulas de jatobá (Hymenaea intermedia Ducke var. adenotricha (Ducke) Lee \& Lang.) (Leguminosaecaesalpinioideae). ACTA Amazônica, v. 34, p. 9-14, 2004.

DO NASCIMENTO, H. H. C.; NOGUEIRA, R. J. M. C.; DA SILVA, E. C.; DA SILVA, M. A. Análise do crescimento de mudas de jatobá (Hymenaea courbaril L.) em diferentes níveis de água no solo. Revista Árvore, v. 35, p. 617-626, 2011.

FARIAS, K. S.; AUHAREK, S. A.; CUNHA-LAURA, A. L.; DE SOUZA, J. M. E.; DAMASCENO-JUNIOR, G. A.; TOFFOLI-KADRI, M. C.; FILIÚ, W. F. O.; DOS SANTOS, E. A.; CHANG, M. R.; CAROLLO, C. A. Adulteration and contamination of comercial sap of Hymenaea Species. Evidence-based Complementary and Alternative Medicine, v. 2017, p. 1-13, 2017.

FREITAS, R. A.; MARTIN, S.; PAULA, R. C.; FEITOSA, J. P. A.; SIERALOWSKI, M. R. Effect of the oxidation level on the thermogravimetric kinetics of on oxidized galactoxyloglucan from Hymenaea courbaril (Jatobá) seeds. Thermochimica Acta, v. 409, p. 41-47, 2004.

INMET (Instituto Nacional de Meteorologia). Banco de Dados Meterológicos para Ensino e Pesquisa. Disponível em: http://www.inmet.gov.br/portal/index.php?r=bdmep/bdm ep. Acesso em: 18 ago. 2017.
LOCOSSELLI, G. M.; SCHÖNGART, J.; CECCANTINI, G. Climate/growth relations and teleconnections for a Hymenaea courbaril (Leguminosae) populations inhabiting the dry forest and karst. Trees, v. 30, p. 1127-1139, 2016.

LUCYSZYN, N.; LUBAMBO, A. F.; MATOS, K. F.; MARVILLA, I.; SOUZA, C. F.; SIERAKOWSKI, M. R. Specific modification of xyloglucan from Hymenaea courbaril seeds. Materials Science and Engineering C., n. 29, p. 552-558, 2009.

MATUDA, T. G.; MARIA NETO, F. Caracterização química parcial de sementes de jatobá-do-cerrado (Hymenaea stigonocarpa Mart.). CiênciaTecnológica de Alimentos, n. 25, p. 353-357, 2005.

MIYAKE, M.; IDE, K.; SASAKI, K.; MATSUKURA, Y.; SSHIJIMA, K.; FUJIWARA, D. Oral administration of Highly Oligomeric Procyanidins of Jatoba reduces the severity of Collagen-Induced Arthritis. Bioscience, Biotechnology and Biochemistry, n. 72, p. 17811788, 2008.

ORSI, P. R.; BONAMIN, F.; SEVERI, J. A.; SANTOS, R. C.; VILEGAS, W.; HIRUMA-LIMA, C. A.; DI STASI, L. C. Hymenaea stigonocarpa Mart. ex Hayne: a Brazilian medicinal plant with gastric and duodenal antiulcer and antidiarrheal effects in experimental rodent models. Journal of Ethnopharmacology, n. 143, p. 81-90, 2012.

SASAKI, K.; MATSUKURA, Y.; SHIJIMA, S.; MIYAKE, M.; FUJIWARA, D.; KONISHI, Y. High-Performance Liquid Chromatographic Purification of oligomeric procyanidins trimers up to nonamers, derived from bark of jatobá (Hymenaea courbari). Bioscience, Biotechnology and Biochemistry, n. 73, p. 12741279, 2009.

SILVA, M. R.; SILVA, M. S.; MARTINS, K. A.; BORGES, S. Utilização tecnológica dos frutos de jatobá-do-cerrado e de jatobá-da-mata na elaboração de biscoitos fontes de fibra alimentar e isentos de açúcares. Ciência Tecnológica de Alimentos, v. 21, p. 176-182, 2001. 